\title{
Continuous renal replacement therapy in children with multiple organ dysfunction syndrome: A case series
}

Yan-lin Zhang ${ }^{1,2}$, Wei-ping Hu',2, Ling-hui Zhou ${ }^{1,2}$, Yin Wang ${ }^{1,2}$, Ao Cheng ${ }^{1,2}$, Si-nan Shao ${ }^{1,2}$, Ling-ling Hong ${ }^{1,2}$, Qiu-yue Chen ${ }^{1,2}$

${ }^{1}$ Department of Nephrology, The First Affiliated Hospital of Xiamen University, Xiamen University, Xiamen 361003, People's Republic of China; ${ }^{2}$ Department of Nephrology, The First Hospital of Xiamen, Fujian Medical University, Xiamen 361003, People's Republic of China

\section{ABSTRACT}

There is a lack of definitive information regarding the precise indications, implementation, and outcomes of continuous renal replacement therapy (CRRT) for the treatment of critically ill children. Six children (three boys, three girls) aged from 3 days to 8 years, all of whom had multiple organ failure, were submitted to bedside CRRT using M60 filter membranes. Modified Port carbonate formula was used and clotting time was maintained between 20 and 30 minutes. Activated partial thromboplastin time was 1.5 - to 2-fold normal. One patient discontinued treatment due to family decision. Marked improvements were seen in the remaining five patients, including normalization of blood urea nitrogen and creatinine levels, stabilization of electrolytes, and improvements in markers of organ function. Of note, one patient (a six-year-old male) underwent the treatment for 241 hours. All five patients were subsequently discharged and recovered uneventfully. CRRT is effective for the management of children who are critically ill due to multiple organ failure.

\section{ARTICLE INFO}

\section{Key words:}

Child; Organ Dysfunction Scores;

Renal Replacement Therapy;

Multiple Organ Failure

Int Braz J Urol. 2014; 40: 846-52

Submitted for publication:

November 05, 2013

Accepted after revision:

April 06, 2014

\section{INTRODUCTION}

Continuous renal replacement therapy (CRRT) is frequently used for the treatment of critically ill children with acute kidney disease (1). Although such treatment can be effective $(2,3)$, there is still a lack of consensus concerning the precise indications, the implementation, and the associated outcomes regarding CRRT in children $(2,4)$. Therefore, we felt compelled to share our experience using this approach for treating critically ill children. Specifically, we describe the treatment and outcomes for a series of six critically ill children, all of whom had multiple organ dysfunction syndrome (MODS).

\section{MATERIALS AND METHODS}

Patients

All children were treated in the pediatric intensive care unit of the First Affiliated Hospital of Xiamen University between February 2008 and June 2011. There were three boys and three girls (Tables 1 and 2 for demographic and clinical 
Table 1 - Demographic and treatment characteristics of critically ill children with multiple organ dysfunction syndrome who received continuous renal replacement therapy.

\begin{tabular}{lcccccc}
\hline Characteristic & Case 1 & Case 2 & Case 3 & Case 4 & Case 5 & Case 6 \\
\hline Age, years & 6 & 7 & 1.5 & 3 days & 4 & 8 \\
Gender & Male & Female & Male & Female & Female & Male \\
Weight, kg & 23 & 13 & 11 & 4 & 10 & 23 \\
Vascular access & RFV & RFV & RFV & RSCV & RFV & RFV \\
Catheter & $8.5 F r$ & $8.5 F r$ & $6.5 F r$ & $6.5 F r$ & $6.5 F r$ & $8.5 F r$ \\
Dialysis filter & M60 & M60 & M60 & M60 & M60 & M60 \\
Anticoagulant & Heparin & Heparin & No heparin & Heparin & No heparin & Fragmin \\
First dose, units & 500 & NA & NA & 100 & NA & 1000 \\
Maintenance dose (units/h) & $104-331$ & 62.5 & NA & $62.5-125$ & NA & $500(4 \mathrm{~h})$ \\
Treatment modality & CVVHDF & CVVHDF & CVVHDF & CVVH & CVVH & CVVHDF \\
Blood flow rate, $\mathrm{mL} / \mathrm{min}$ & 80 & 75 & 30 & 30 & 60 & 60 \\
Replacement fluid flow rate, $\mathrm{mL} / \mathrm{h}$ & 600 & 1000 & 300 & 500 & 800 & 400 \\
Dialysate flow rate, $\mathrm{mL} / \mathrm{h}$ & 300 & 500 & 200 & 0 & 0 & 300 \\
Fluid removal rate, $\mathrm{mL} / \mathrm{h}$ & $50-100$ & $0-50$ & $0-40$ & $20-60$ & 0 & 0 \\
Duration of CRRT, h & 241 & 86 & 12 & 52 & 5 & 6 \\
\hline
\end{tabular}

CRRT: continuous renal replacement therapy; CVVHDF: continuous venovenous hemofiltration with dialysis; CVVH: continuous venovenous hemofiltration; NA: not available; RFV: right femoral vein; RSCV: right subclavian vein.

characteristics). All suffered from MODS (Table-3) due to sepsis and acute rhabdomyolysis syndrome (Case 1), septic shock (Cases 2, 3, and 6), neonatal asphyxia and meconium aspiration syndrome (Case 4), or hemorrhagic shock, ureter and bladder rupture, and crush syndrome (caused by a car accident) (Case 5).

\section{Treatment}

A PRISMA bedside CRRT machine (Hospal, Lyon, France) and M60 AN69 membrane filters were used for all patients. Double- or single-lumen veno-venous hemodialysis catheters were inserted via the right femoral or subclavian vein. The replacement fluid (modified Port carbonate formula) was infused using the post-dilution method. For anticoagulation, patients received heparin or low molecular weight heparin (note: some patients did not receive hepa- rin). Clotting time was maintained between 20 and 30 minutes and tested using the capillary tube method. Activated partial thromboplastin time was 1.5-2.0 times normal.

\section{Outcomes}

One patient (case 5) discontinued treatment due to a family decision and eventually died due to circulatory failure and electrolyte disturbance. Dramatic improvements were seen in clinical measures after treatment in all other patients (Table-2). Of note, blood urea nitrogen (BUN) and creatinine $(\mathrm{Cr})$ levels were normalized, electrolyte and acid-base balance was stabilized, and indicators of organ function were markedly improved. These patients were subsequently discharged.

Below, we describe two cases of particular interest in more detail. 


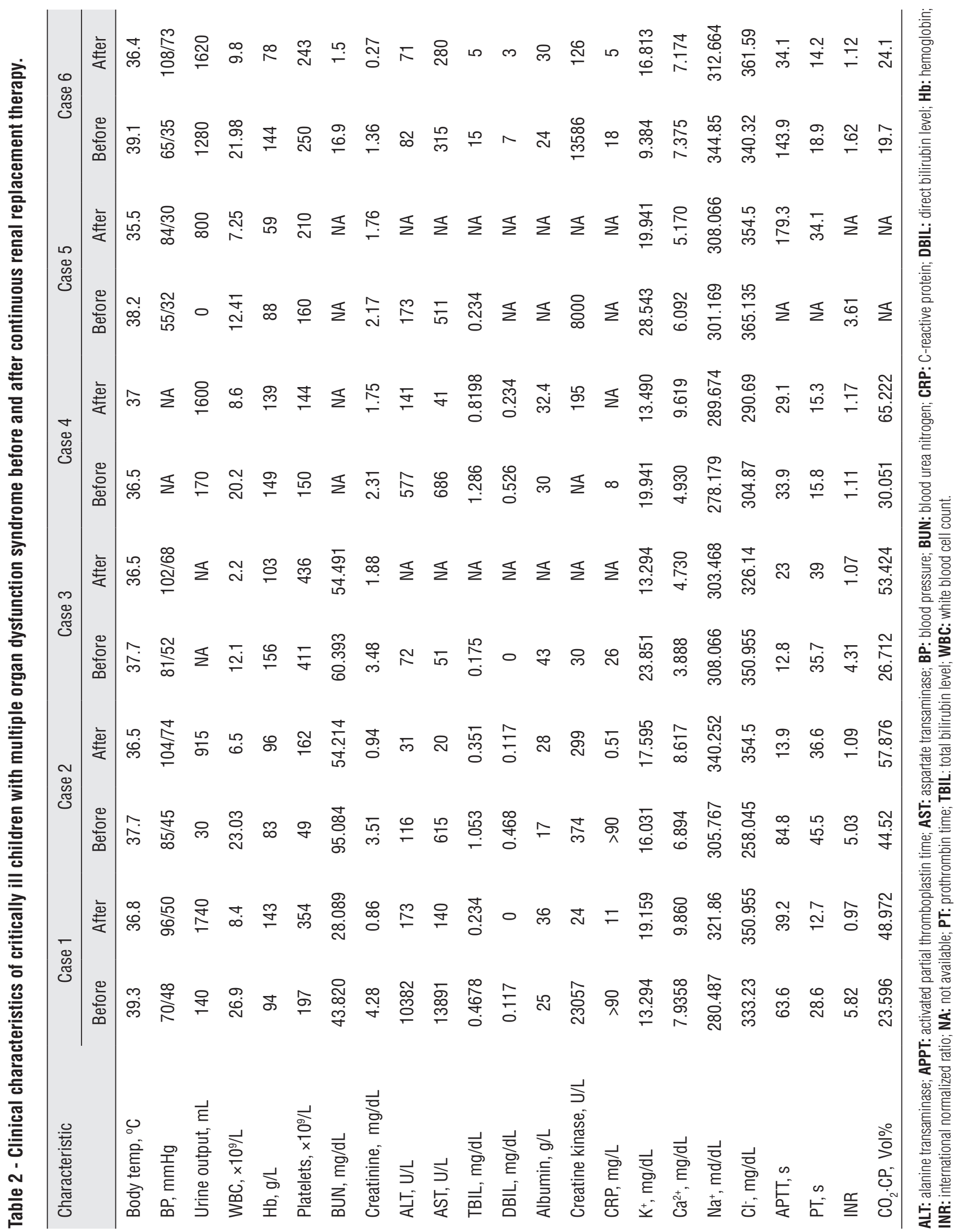


Table 3 - Summary of multiple organ dysfunction scores for each critally ill child who received continuous renal replacement therapy.

\begin{tabular}{lcccccc}
\hline Case & Respiration & Kidney & Liver & Blood System & $\begin{array}{c}\text { Nervous } \\
\text { System }\end{array}$ & Total \\
\hline 1 & 2 & 2 & 1 & 1 & 2 & 8 \\
2 & 2 & 2 & 2 & 3 & 2 & 11 \\
3 & 2 & 2 & 1 & 2 & 2 & 9 \\
4 & 2 & 2 & 2 & 2 & 2 & 10 \\
5 & 2 & 2 & 2 & 1 & 2 & 9 \\
6 & 2 & 2 & 1 & 1 & 2 & 8 \\
\hline
\end{tabular}

Case 1

Case 1 was a six-year-old boy who was admitted on 9 January 2011 with a two-day history of non-projectile vomiting and diarrhea, yellow watery stool, and fever. He had also experienced a convulsion. After admission, the patient was diagnosed with toxic bacterial enteritis, sepsis, MODS (acute kidney injury [AKI], stress ulcer, infection-induced toxic encephalopathy, early disseminated intravascular coagulation, acute myocardial injury, acute liver injury, acute rhabdomyolysis), hyponatremia, metabolic acidosis, and hypoalbuminemia. The patient was experiencing blurred consciousness on admission and suffered a convulsion the day after admission, with a concomitant sustained decrease in blood pressure and urine output. Blood testing revealed abnormal coagulation, metabolic acidosis, electrolyte imbalance, and progressively increased BUN and Cr levels. Serum Cr peaked at $378 \mu \mathrm{mol} / \mathrm{L}$, while creatine kinase was $23057 \mathrm{U} / \mathrm{L}$. The patient underwent CRRT from 11-20 January, and regained consciousness on January 16th. His liver and renal function gradually improved, and blood pressure and urine output normalized. The patient underwent CRRT for 241 hours and was hospitalized for 33 days before discharge. After discharge, the patient was followed-up once-monthly for six months. His development was normal and there was no relapse of symptoms/biochemical abnormalities.

\section{Case 4}

Case 4 was a three-day-old female neonate who was transferred to the neonatal intensive care unit on 16 February 2008. She had a history of no crying, poor responsiveness, and anuria. Diagnoses after admission were neonatal asphyxia and meconium aspiration syndrome, neonatal AKI, MODS (heart, liver, kidney, brain), neonatal hypoxic encephalopathy, and severe electrolyte imbalance. Her serum Cr was $204 \mu \mathrm{mol} / \mathrm{L}$ on admission and $429 \mu \mathrm{mol} / \mathrm{L}$ on the day after admission. CRRT was initiated. Her electrolytes and urine output gradually normalized. The patient underwent bedside CRRT for 52 hours and was hospitalized for 20 days before discharge. After discharge, the patient was followed-up once-monthly for six months. Her development was normal and there was no relapse of symptoms or biochemical abnormalities.

\section{DISCUSSION}

Herein, we have summarized our experience treating six children with MODS using CRRT. Of the five children who received full treatment, all recovered completely, thus supporting the effectiveness of CRRT for the management of children who are critically ill due to MODS.

Two cases in our series warrant further discussion, Cases 1 and 4. Case 1 was a six-year-old boy, whereas Case 4 was a three-day-old female neonate; both were in critical condition and would have died without intervention. Case 1 was unique for the duration of CRRT (241 hours), whereas Case 4 was unique because there have been few reports of survival in neonates with MODS af- 
ter CRRT $(3,5,6)$. In both of these cases, and indeed in general, we believe that early intervention with CRRT may have inhibited the cytokine cascade/ systematic inflammatory response and the associated inflammatory injury (7). Clearly, the amelioration of uremia and fluid overload likely contributed to the efficacy of treatment. According to our review of the literature, CRRT for 241 hours is very rare. This duration of treatment was necessary to stabilize the patient's internal environment, provide nutritional/metabolic support, and allow for the implementation of other supportive treatment. For this patient, an AN69 membrane filter M60 with good biocompatibility, high permeability, and a strong adsorption capacity was used. The area of the filter was relatively large $\left(0.6 \mathrm{~m}^{2}\right)$. The membrane, which can also be used under hypotension, causes a weak activation of complement and leukocytes, and has a small impact on hemodynamics. The high-permeability of this membrane may help to improve the anti-inflammatory cytokine to proinflammatory cytokine ratio, down-regulate the body's inflammatory response, and ameliorate the systemic inflammatory response (8).

One of the keys to early intervention in the neonate was placement of a central venous catheter by an experienced anesthesiologist. The smallest M60 filter was used and the tubing was pre-filled with whole blood. Further, at the beginning of CRRT, the blood flow rate was low, thus preventing a subsequent drop in blood pressure (9). Attention was also paid to body heat preservation and heating the vascular access point. This case highlights that, in addition to having the appropriate equipment, inter-departmental cooperation can be important for successful CRRT. Staff in neonatal/pediatric intensive care units should not hesitate to seek assistance from staff in other departments who may have appropriate expertise in central vein cannulation and CRRT.

We also believe it is worthwhile to summarize the key technical principles and requirements for successful implementation of CRRT in children. Due to the physiological and anatomical characteristics of children, including low body weight, small blood flow volume, and hemodynamic instability, filters and tubing with a low pre-filled vo- lume should be used to reduce the blood volume in extracorporeal circulation and help ameliorate the decrease in effective circulating blood volume. Filters with a high molecular weight polymer membrane, high permeability, good biocompatibility, and a small impact on the coagulation system should be selected. The use of AN69 membranes has been linked to bradykinin release syndrome among patients who are acidotic or taking angiotensin converting enzyme inhibitors $(10,11)$. Alternative membranes should be used in such cases. Generally, the blood volume in extracorporeal circulation should be maintained at $<10 \%$ of body weight, ie, $<30 \mathrm{~mL}$ in neonates, $<50 \mathrm{~mL}$ in infants, and $<100 \mathrm{~mL}$ in children. For neonates weighing $<2.5 \mathrm{~kg}$, tubing can be pre-filled with plasma, whole blood, or 5\% albumin (12).

Establishing vascular access is a key factor for successful CRRT in children. The most frequently used veins in children are the femoral and internal jugular. For operative easiness and to help maintain stable blood flow, veno-venous double-lumen hemodialysis catheters are often used. Two single-lumen venous hemodialysis catheters can be used in infants. Recommended catheters by age are: $<6$ months, 4-5F single-lumen; 6-12 months, 5-7F double-lumen; 1-3 years, 8-9F double-lumen; and $>3$ years, 8-12F double-lumen (13).

Determining the therapeutic dose for continuous veno-venous hemofiltration in critically ill children remains a challenge. Individual doses should be determined according to the disease conditions, taking into consideration metabolic state, fluid volume, and duration of dialysis (14). Transmembrane pressure should be monitored and maintained at $<200 \mathrm{mmHg}$ (note: a transmembrane pressure $>250 \mathrm{mmHg}$ may indicate clotting in the filter) (15). Particular attention should be paid to warming the replacement fluid. The recommended flow rates are as follows. Blood flow: $30 \mathrm{~mL} / \mathrm{min}$ in neonates; $30-40 \mathrm{~mL} / \mathrm{min}$ in infants/young children; $50-75 \mathrm{~mL} / \mathrm{min}$ in children weighing $<20 \mathrm{~kg}$; and $75-100 \mathrm{~mL} / \mathrm{min}$ in children weighing $>20 \mathrm{~kg}$. Ultrafiltration rate: $8-10 \mathrm{~mL} / \mathrm{min} / \mathrm{m}^{2}$ in neonates/ infants; $8-15 \mathrm{~mL} / \mathrm{min} / \mathrm{m}^{2}$ in children (note: daily fluid input/output, cardiac function, and edema should be considered). Dialysates: $15-20 \mathrm{~mL} / \mathrm{min} /$ $\mathrm{m}^{2}$ in neonates/infants/children (16). 
We believe there are a number of key factors that should be taken into account when considering CRRT in cases similar to those described herein. For cases involving AKI or acute renal failure, particular attention should be paid to water and sodium retention and blood nitrogen levels, all of which have a significant impact on the treatment and survival of infants and young children (17), as well as the speed of progress. The characteristics of systemic disease are also an important factor (18). CRRT rescue was successful in the 5 patients described herein who completed treatment. We believe that several reasons underlie this high rate of treatment success. First, the decision to treat with CRRT was made early. Our approach is consistent with that advocated by Wolf et al., who suggest that the survival rate following extracorporeal circulation can be improved if treatment is commenced as soon as possible (19). Second, CRRT allows for correction of water and sodium retention and electrolyte imbalances in a timely manner, facilitating the effectiveness of other treatments (20). Finally, we must reiterate, that involvement and cooperation between a multi-disciplinary team is of paramount importance so that appropriate rescue of respiratory failure, use of breathing machines, infection prevention and treatment, and cardiovascular support can be accomplished. With regards to when to withdraw treatment, we believe that CCRT can be halted once water and sodium retention subsides, urine output returns to normal, and renal function recovers. To this end, it is worth noting that body water load and urine output are directly related to survival in children $(4,21)$.

Over the last few decades, CRRT has become increasingly used in pediatrics, particularly for treating critical illness, such as severe acute renal failure and MODS (9). With further study into the mechanisms underlying the efficacy of CRRT and technological advances, the effectiveness of CRRT for treating critically ill children can only improve. We hope the sharing of our experience with this treatment will help facilitate such improvement.

\section{ABBREVIATIONS}

AKI = acute kidney injury

$\mathrm{ALT}=$ alanine transaminase
APPT $=$ activated partial thromboplastin time

AST $=$ aspartate transaminase

$\mathrm{BP}=$ blood pressure

BUN = blood urea nitrogen

$\mathrm{Cr}=$ creatinine

$\mathrm{CRP}=\mathrm{C}$-reactive protein

CRRT = continuous renal replacement therapy

CVVHDF = continuous venovenous hemofiltra-

tion with dialysis

CVVH = continuous venovenous hemofiltration

DBIL = direct bilirubin level

$\mathrm{Hb}=$ hemoglobin

INR = international normalized ratio

MODS = multiple organ dysfunction syndrome

$\mathrm{NA}=$ not available

$\mathrm{PT}=$ prothrombin time

$\mathrm{RFV}=$ right femoral vein

RSCV = right subclavian vein

TBIL $=$ total bilirubin level

WBC $=$ white blood cell count

\section{CONFLICT OF INTEREST}

None declared.

\section{REFERENCES}

1. Sutherland SM, Alexander SR. Continuous renal replacement therapy in children. Pediatr Nephrol. 2012; 27: 2007-16.

2. Goldstein SL. Continuous renal replacement therapy: mechanism of clearance, fluid removal, indications and outcomes. Curr Opin Pediatr. 2011; 23: 181-5.

3. Hayes LW, Oster RA, Tofil NM, Tolwani AJ. Outcomes of critically ill children requiring continuous renal replacement therapy. J Crit Care. 2009; 24: 394-400.

4. Askenazi DJ, Goldstein SL, Koralkar R, Fortenberry J, Baum M, Hackbarth R, et al. Continuous renal replacement therapy for children $\leq 10 \mathrm{~kg}$ : a report from the prospective pediatric continuous renal replacement therapy registry. J Pediatr. 2013; 162: 587-592.e3.

5. Goldstein SL, Somers MJ, Baum MA, Symons JM, Brophy PD, Blowey D, et al. Pediatric patients with multiorgan dysfunction syndrome receiving continuous renal replacement therapy. Kidney Int. 2005; 67: 653-8.

6. Ponikvar R, Kandus A, Urbancic A, Kornhauser AG, Primozic J, Ponikvar JB. Continuous renal replacement therapy and plasma exchange in newborns and infants. Artif Organs. 2002; 26: 163-8. 
7. Splendiani G, Mazzarella V, Cipriani S, Zazzaro D, Casciani CU. Continuous renal replacement therapy: our experience in intensive care unit. Ren Fail. 2001; 23: 259-64.

8. Mu TS, Palmer EG, Batts SG, Lentz-Kapua SL, Uyehara-Lock $\mathrm{JH}$, Uyehara CF. Continuous renal replacement therapy to reduce inflammation in a piglet hemorrhage-reperfusion extracorporeal membrane oxygenation model. Pediatr Res. 2012; 72: 249-55.

9. Lee CY, Yeh $\mathrm{HC}$, Lin CY. Treatment of critically ill children with kidney injury by sustained low-efficiency daily diafiltration. Pediatr Nephrol. 2012; 27: 2301-9.

10. Brophy PD, Mottes TA, Kudelka TL, McBryde KD, Gardner JJ, Maxvold NJ, et al. AN-69 membrane reactions are $\mathrm{pH}-$ dependent and preventable. Am J Kidney Dis. 2001; 38: 1738.

11. Tielemans C, Madhoun P, Lenaers M, Schandene L, Goldman $\mathrm{M}$, Vanherweghem JL. Anaphylactoid reactions during hemodialysis on AN69 membranes in patients receiving ACE inhibitors. Kidney Int. 1990; 38: 982-4.

12. Kendirli T, Ekim M, Ozçakar ZB, Yüksel S, Acar B, OztürkHiişmi $B$, et al. Renal replacement therapies in pediatric intensive care patients: experiences of one center in Turkey. Pediatr Int. 2007; 49: 345-8.

13. Hackbarth R, Bunchman TE, Chua AN, Somers MJ, Baum M, Symons JM, et al. The effect of vascular access location and size on circuit survival in pediatric continuous renal replacement therapy: a report from the PPCRRT registry. Int J Artif Organs. 2007; 30: 1116-21.
14. Sebestyen JF, Warady BA. Advances in pediatric renal replacement therapy. Adv Chronic Kidney Dis. 2011; 18: 376-83.

15. Baldwin I. Is there a need for a nurse emergency team for continuous renal replacement therapy? Contrib Nephrol. 2007;156:191-6.

16. Bunchman TE, Brophy PD, Goldstein SL. Technical considerations for renal replacement therapy in children. Semin Nephrol. 2008; 28: 488-92.

17. Askenazi DJ, Koralkar R, Hundley HE, Montesanti A, Patil N, Ambalavanan N. Fluid overload and mortality are associated with acute kidney injury in sick near-term/term neonate. Pediatr Nephrol. 2013; 28: 661-6.

18. Bock KR. Renal replacement therapy in pediatric critical care medicine. Curr Opin Pediatr. 2005; 17: 368-71.

19. Wolf MJ, Chanani NK, Heard ML, Kanter KR, Mahle WT. Early renal replacement therapy during pediatric cardiac extracorporeal support increases mortality. Ann Thorac Surg. 2013; 96: 917-22.

20. Boschee ED, Cave DA, Garros D, Lequier L, Granoski DA, Guerra GG, et al. Indications and outcomes in children receiving renal replacement therapy in pediatric intensive care. J Crit Care. 2014; 29: 37-42.

21. Sutherland SM, Zappitelli M, Alexander SR, Chua AN, Brophy $\mathrm{PD}$, Bunchman TE, et al. Fluid overload and mortality in children receiving continuous renal replacement therapy: the prospective pediatric continuous renal replacement therapy registry. Am J Kidney Dis. 2010; 55: 316-25.

Correspondence address: Yan-lin Zhang, MD

Department of Nephrology,

The First Affiliated Hospital of Xiamen University and The First Hospital of Xiamen, Fujian Medical University, Xiamen 361003, People's Republic of China Fax: + 86592 213-0076 E-mail: zyl59@139.com 